\title{
EIGENTIME IDENTITY FOR ONE-DIMENSIONAL DIFFUSION PROCESSES
}

\author{
LI-JUAN CHENG, ${ }^{* *}$ Zhejiang University of Technology and Beijing Normal University \\ YONG-HUA MAO, ${ }^{* * *}$ Beijing Normal University
}

\begin{abstract}
The eigentime identity for one-dimensional diffusion processes on the halfline with an entrance boundary at $\infty$ is obtained by using the trace of the deviation kernel. For the case of an exit boundary at $\infty$, a similar eigentime identity is presented with the aid of the Green function. Explicit equivalent statements are also listed in terms of the strong ergodicity or the uniform decay for diffusion processes.
\end{abstract}

Keywords: Hitting time; lifetime; eigentime identity; strong ergodicity; uniform decay; eigenvalue

2010 Mathematics Subject Classification: Primary 60J60; 47A75

\section{Introduction and main results}

In this paper we study the eigentime identity for one-dimensional nonsingular diffusion processes on the halfline $\mathbb{R}_{+}:=[0,+\infty)$ with a reflecting boundary at 0 , whose definition is generalized from finite Markov chains (cf. [1, Chapter 3]) to this setting. Roughly speaking, we are looking for some typical quantities in terms of some random times to characterize the behavior of one-dimensional diffusion processes. The readers are urged to refer to [13] and [14] for the background and the present status of the study on this topic. We would like to indicate that the eigentime identity raises the study of more general passage times, e.g. lifetime, which is further applied to strong stationary times for the diffusion case; see our recent result [3] for details.

Let $a$ be a positive and continuous function on $[0,+\infty)$ and $b$ be a locally integrable function on $[0,+\infty)$. Consider the diffusion operator

$$
L:=a(x) \frac{\mathrm{d}^{2}}{\mathrm{~d} x^{2}}+b(x) \frac{\mathrm{d}}{\mathrm{d} x},
$$

with diffusion coefficient $a$ and drift $b$ acting on a domain of functions denoted by $\mathscr{D}(L)$ subject to some appropriate smoothness and boundary conditions (see, e.g. [2, pp. 15-17]). Define $c(x)=\int_{0}^{x} b(u) / a(u) \mathrm{d} u$ for all $x>0$, and $\mu(\mathrm{d} x)=a(x)^{-1} \mathrm{e}^{c(x)} \mathrm{d} x$. Then, it is well known that $(L, \mathscr{D}(L))$ is a nonpositive definite, and self-adjoint operator on $L^{2}(\mu)$. Adopting the standard Feller's notation (see [7], [10]) for a one-dimensional diffusion operator, $\mu(\mathrm{d} x)$ and $s(x):=\int_{0}^{x} \mathrm{e}^{-c(u)} \mathrm{d} u$ are called the speed measure and the scale function, respectively.

Let $X=\left(X_{t}: t \geq 0\right)$ be a diffusion process generated by $L$ on the halfline $[0, \infty)$ with reflecting boundary 0 . We investigate the stated problem according to the type of boundary $\infty$.

Received 26 April 2013; revision received 15 February 2014.

* Postal address: Department of Applied Mathematics, Zhejiang University of Technology, Hangzhou 310023, China.

** Email address: chenglj@mail.bnu.edu.cn

*** Email address: maoyh@mail.bnu.edu.cn 
We first consider the process $\left(X_{t}\right)$ with $\infty$ the reflecting (Neumann) boundary, i.e. the process starting from any point will certainly come back. Define

$$
R=\int_{0}^{\infty} \mu((0, x)) \mathrm{d} s(x) \text { and } S=\int_{0}^{\infty} s(x) \mu(\mathrm{d} x) .
$$

Actually, by Feller's terminology (see [5], [6]), we will consider $\infty$ as an entrance boundary, where boundary $\infty$ is called entrance if $R=\infty$ and $S<\infty$, or, equivalently, $S<$ $\infty, \mu((0, \infty))<\infty$, and $s(\infty)=\infty$. Let $\pi(\mathrm{d} x)=\mu(\mathrm{d} x) / Z$, where $Z:=\mu([0, \infty))<\infty$. Then $L$ is a self-adjoint operator in $L^{2}(\pi)$. Denote the essential spectrum of $L$ in $L^{2}(\pi)$ by $\sigma_{\text {ess }}(L)$. We write $\lambda \in \sigma_{\text {ess }}(L)$ if, for any $\varepsilon>0$, the closure of the range of $\mathbf{1}_{(\lambda-\varepsilon, \lambda+\varepsilon)}(L)$ is infinite-dimensional, where $\mathbf{1}$ is the indicator function. For a deeper discussion of the essential spectrum, see [18]. When $\sigma_{\text {ess }}(L)=\varnothing$, denote all the eigenvalues of $-L$ in $L^{2}(\pi)$ by

$$
0=\lambda_{0}<\lambda_{1}<\lambda_{2}<\cdots .
$$

Let $H_{y}:=\inf \left\{t \geq 0, X_{t}=y\right\}$ be the hitting time of $y$. Define the average hitting time by

$$
T=\iint \mathbb{E}_{x} H_{y} \pi(\mathrm{d} x) \pi(\mathrm{d} y) .
$$

The eigentime identity for $\left(X_{t}\right)$ is presented as follows.

Theorem 1.1. Let $L$ be defined as in (1.1) with positive and continuous function a and locally integrable function $b$. Assume that $R=\infty$ and $S<\infty$. Then $\sigma_{\mathrm{ess}}(L)=\varnothing$. Moreover, for the one-dimensional diffusion process $\left(X_{t}\right)$ generated by $L$ with reflecting boundary 0 , the eigentime identity is

$$
T=\sum_{n=1}^{\infty} \lambda_{n}^{-1}<\infty
$$

where $\mathbb{E}_{x}$ stands for the expectation taken for the underlying process starting from $x$.

Before moving on, let us make some comments on Theorem 1.1.

(a) The average hitting time is closely related to time-asymptotic behaviors of the diffusion process; see [1, Chapter 4] for the corresponding results for finite reversible Markov chains.

(b) From Theorem 2.1 below, $T<\infty$ implies the existence of a spectral gap of $-L$ in $L^{\infty}(\pi)$, which measures the rate of the strong ergodicity.

(c) The method to prove this result is based on the fact that the trace of the deviation kernel with respect to $\pi$ equals the average hitting time (see Lemma 2.1 below).

We now turn to the case with $\infty$ as the absorbing (Dirichlet) boundary, i.e. the diffusion process starting from any point will be certainly absorbed at $\infty$. In fact, we will study $\infty$ as an exit boundary (see [5], [6]), where boundary $\infty$ is called exit if $R<\infty$ and $S=\infty$, or equivalently, $R<\infty, \mu((0, \infty))=\infty$, and $s(\infty)=\int_{0}^{\infty} \mathrm{e}^{-c(u)} \mathrm{d} u<\infty$.

It is already known that when $R<\infty$, the corresponding diffusion process is not unique (see, e.g. [8] and the references therein). We are interested in the minimal process $\left(X_{t}\right)$. (The minimal process is a Markov process on $([0, \infty], \mathcal{B}([0, \infty]))$ with $\{\tilde{P}(t, x, \mathrm{~d} y)\}$ as transition 
probabilities, where $\tilde{P}(t, x, B)=P(t, x, B)$ if $x \in \mathbb{R}_{+}, B \in \mathcal{B}\left(\mathbb{R}_{+}\right) ; \tilde{P}(t, x,\{\infty\})=$ $1-P\left(t, x, \mathbb{R}_{+}\right)$if $x \in \mathbb{R}_{+} ; \tilde{P}\left(t, \infty, \mathbb{R}_{+}\right)=0, \tilde{P}(t, \infty,\{\infty\})=1$, and $P(t, x, \mathrm{~d} y)$ is the subprobability associated with $L$.) Define the lifetime

$$
\zeta=\lim _{x \rightarrow \infty} H_{x}
$$

When the essential spectrum of $L$, with respect to $L^{2}(\mu)$, satisfies $\sigma_{\text {ess }}(L)=\varnothing$, we denote all eigenvalues of $-L$ by

$$
0<\lambda_{1}<\lambda_{2}<\cdots
$$

The eigentime identity for the minimal diffusion process is presented in the following theorem.

Theorem 1.2. Let $L$ be defined as in (1.1) with positive and continuous function a and locally integrable function $b$. Assume that $R<\infty$ and $S=\infty$. Then $\sigma_{\mathrm{ess}}(L)=\varnothing$. Moreover, for the one-dimensional minimal diffusion process $\left(X_{t}\right)$ generated by $L$ with reflecting boundary 0 , the eigentime identity reads as

$$
\mathbb{E}_{0} \zeta=\sum_{n=1}^{\infty} \lambda_{n}^{-1}<\infty
$$

We will also give some notes on this theorem.

(a) We would like to use the approximation processes of [3] to give the distribution of the lifetime, but the main obstacle is to clarify the spectral relationship between the original process and the approximating processes. The eigentime identity established in Theorem 1.2 helps us to solve this problem (see [3, Lemma 2.7]).

(b) It is well known that $R$ is just the expectation of the first passage time of $\left(X_{t}\right)$ from 0 to $\infty$, i.e. $R=\mathbb{E}_{0} \zeta<\infty$ (see [8]), which implies the uniform decay of the process; see Theorem 3.1 below.

(c) Compared with earlier works (see [13], [14], and the references therein), the equation for the Laplace transform of the hitting time will become a powerful tool in order to deal with our setting.

The rest of this paper is organized as follows. In the next section we will present the proof of Theorem 1.1 first, and then illustrate the relationship among $T$, eigenvalues of $-L$, and the strong ergodicity. In Section 3 we will complete the proof of Theorem 1.2 and estimate the uniform decay rate by the spectrum in $L^{2}(\mu)$.

\section{Entrance boundary}

In this section we are committed to the study of the diffusion process $\left(X_{t}\right)$ with $\infty$ the entrance boundary generated by $L$.

\subsection{Proof of Theorem 1.1}

Assume that $\left(P_{t}, t \geq 0\right)$ is the Markov semigroup associated with $L$ and $p(t, x, y)$ is the transition density of the ergodic process $\left(X_{t}\right)$ relative to the invariant probability measure $\pi$, i.e.

$$
P(t, x, \mathrm{~d} y)=p(t, x, y) \pi(\mathrm{d} y) .
$$


Remark that $p(t, x, y)$ can be chosen to be jointly continuous in $(t, x, y) \in \mathbb{R}_{+}^{3}$ (cf. [7, Section 4.11]). Our method is based on the deviation kernel, which is defined by

$$
d(x, y):=\int_{0}^{\infty}(p(t, x, y)-1) \mathrm{d} t, \quad x, y \in[0, \infty) .
$$

By [11, Theorem 2.1], the function $d(x, y)$ exists and is finite for all $x, y \in \mathbb{R}_{+}$if and only if $\int_{0}^{\infty} \mathbb{E}_{x} H_{y} \pi(\mathrm{d} x)<\infty$ for some $y \in \mathbb{R}_{+}$. It is easy to see that $S<\infty$ implies that $d(x, y)$ is well defined. The following lemma, in which we present some important properties of the deviation kernel, is essential to the proof of Theorem 1.1.

Lemma 2.1. Under the same conditions as in Theorem 1.1, the function $d(x, y)$ exists and is finite for all $x, y \in \mathbb{R}_{+}$, and

$$
\int_{0}^{\infty} d(y, y) \pi(\mathrm{d} y)=T<\infty .
$$

Proof. Also from [11, Theorem 2.1], we know that $d(y, y)=\int_{0}^{\infty} \mathbb{E}_{x} H_{y} \pi(\mathrm{d} x)$ for any $y \in$ $\mathbb{R}_{+}$. So, it suffices for us to prove that ' $T<\infty$ '. We now turn to show that ' $S<\infty$ ' implies that ' $T<\infty$ '.

Actually, ' $S<\infty$ ' is equivalent to ' $T<\infty$ '. It is well known (see, e.g. [8, Chapter 15]) that

$$
\mathbb{E}_{x} H_{y}= \begin{cases}\int_{y}^{x} \mu((t, \infty)) \mathrm{d} s(t), & x>y, \\ \int_{x}^{y} \mu((0, t)) \mathrm{d} s(t), & x \leq y .\end{cases}
$$

By this and the fact that $\pi(\mathrm{d} x)=(1 / Z) \mu(\mathrm{d} x)$, where $Z=\mu([0, \infty))<\infty$, we have

$$
\begin{aligned}
T= & \iint \mathbb{E}_{x} H_{y} \pi(\mathrm{d} x) \pi(\mathrm{d} y) \\
= & \iint_{x>y} \mathbb{E}_{x} H_{y} \pi(\mathrm{d} x) \pi(\mathrm{d} y)+\iint_{x \leq y} \mathbb{E}_{x} H_{y} \pi(\mathrm{d} x) \pi(\mathrm{d} y) \\
= & \frac{1}{Z^{2}} \iint_{x>y} \mu(\mathrm{d} x) \mu(\mathrm{d} y) \int_{y}^{x} \mu((t,+\infty)) \mathrm{d} s(t) \\
& +\frac{1}{Z^{2}} \iint_{x \leq y} \mu(\mathrm{d} x) \mu(\mathrm{d} y) \int_{x}^{y} \mu((0, t)) \mathrm{d} s(t) \\
= & \frac{1}{Z} \int \mu((0, t]) \mu([t, \infty)) \mathrm{d} s(t) .
\end{aligned}
$$

In addition, $S=\int \mu([x, \infty)) \mathrm{d} s(x)$. It is easy to see that $T \leq S$. When $T<\infty$, we choose $t_{0}>0$ such that $\mu\left(\left[0, x_{0}\right]\right) / Z>\frac{1}{2}$. Then,

$$
\infty>T \geq \frac{1}{Z} \int_{\left[x_{0},+\infty\right)} \mu((0, x]) \mu([x, \infty)) \mathrm{d} s(x)>\frac{1}{2} \int_{\left[x_{0},+\infty\right)} \mu([x, \infty)) \mathrm{d} s(x)
$$

and $\int_{\left(0, x_{0}\right)} \mathrm{d} s(x) \mu([x, \infty))<\infty$, which immediately implies that $S<\infty$.

Our initial step is to show that under the assumption of Theorem 1.1, $P_{t}$ is a Hilbert-Schmidt operator on $L^{2}(\pi)$, which is the case if the following Hilbert-Schmidt norm is finite:

$$
\left\|P_{t}\right\|_{H S}^{2}=\sum_{i=1}^{\infty}\left\|P_{t} e_{i}\right\|^{2}
$$

where $\|\cdot\|$ is the norm in $L^{2}(\pi)$ and $\left\{e_{i}\right\}$ is an orthonormal basis of $L^{2}(\pi)$. 
Lemma 2.2. Assume $\infty$ is an entrance boundary. Then $P_{t}$ is a Hilbert-Schmidt operator in $L^{2}(\pi)$ for each $t>0$. Thus, $\sigma_{\mathrm{ess}}(L)=\varnothing$.

Proof. We should check the monotonicity of $p(t, x, x)$ first. Select $t, t_{0} \in(0, \infty)$ such that $t_{0}<t$. By the contraction of $P_{t}$ under $L^{2}$-norm $\|\cdot\|$, we obtain

$$
\begin{aligned}
p(t, x, x) & =\int p^{2}\left(\frac{t}{2}, x, y\right) \pi(\mathrm{d} y) \\
& =\int\left(P_{\left(t-t_{0}\right) / 2} p\left(\frac{t_{0}}{2}, x, \cdot\right)(y)\right)^{2} \pi(\mathrm{d} y) \\
& =\left\|P_{\left(t-t_{0}\right) / 2} p\left(\frac{t_{0}}{2}, x, \cdot\right)\right\|^{2} \\
& \leq\left\|p\left(\frac{t_{0}}{2}, x, \cdot\right)\right\|^{2} \\
& =p\left(t_{0}, x, x\right),
\end{aligned}
$$

where the second equality comes from the property that $P_{t / 2}(x, \mathrm{~d} y)=P_{\left(t-t_{0}\right) / 2} P_{t_{0} / 2}(x, \mathrm{~d} y)$. Therefore, $p(t, x, x)$ is decreasing in $t \in(0, \infty)$.

Let $\phi(t):=\int(p(t, x, x)-1) \pi(\mathrm{d} x)=\int p(t, x, x) \pi(\mathrm{d} x)-1$.

Due to the ergodicity of the process, $p(t, x, y) \rightarrow 1$ as $t \rightarrow \infty$, for all $x, y \in \mathbb{R}_{+}$, which, together with (2.2), implies that

$$
p(t, x, x)-1 \geq 0 \quad \text { for any } t>0 \text { and } x \in \mathbb{R}_{+} .
$$

By this and (2.1), we conclude that

$$
\begin{aligned}
\int_{[0, \infty)} \phi(t) \mathrm{d} t & =\int_{0}^{\infty} \mathrm{d} t \int_{0}^{\infty}(p(t, x, x)-1) \pi(\mathrm{d} x) \\
& =\int_{0}^{\infty} \int_{0}^{\infty}(p(t, x, x)-1) \mathrm{d} t \pi(\mathrm{d} x) \\
& =T \\
& <\infty
\end{aligned}
$$

where the second equality is due to Fubini's equation for nonnegative measurable functions. Then, $\phi(t)<\infty$ almost everywhere (a.e.) $t \in(0, \infty)$. By the monotonicity of $\phi$, we obtain $\phi(t)<\infty$ for all $t \in(0, \infty)$. Hence, $\int p(t, x, x) \pi(\mathrm{d} x)=\phi(t)+1<\infty$ for all $t \in(0, \infty)$. Fix $t>0$, to prove that $P_{t}$ is a Hilbert-Schmidt operator we need to show that $p(t, x, y) \in$ $L^{2}(\pi \times \pi)$. In fact, since $p(t, x, y)$ is symmetric in $x, y$ due to the reversibility of $P_{t}$ in $L^{2}(\pi)$, then by the Markov property, we have

$$
\iint p(t, x, y)^{2} \pi(\mathrm{d} x) \pi(\mathrm{d} y)=\int P(t, x, \mathrm{~d} y) p(t, y, x) \pi(\mathrm{d} x)=\int p(2 t, x, x) \pi(\mathrm{d} x)<\infty .
$$

Therefore, $\sigma_{\mathrm{ess}}\left(P_{t}\right)=\varnothing$, which implies that $\sigma_{\mathrm{ess}}(L)=\varnothing$ by the spectral mapping theorem (see [4, Corollary 2.37]).

When $\sigma_{\text {ess }}(L)=\varnothing$, let $f^{(n)}$ be an eigenfunction corresponding to $\lambda_{n}$ such that $\left\{f^{(n)}: n \geq 0\right\}$ is an orthonormal basis in $L^{2}(\pi)$. We want to prove Theorem 1.1 by using the spectral expression of the transition density. 
Proof of Theorem 1.1. From Lemma 2.2, $\sigma_{\mathrm{ess}}(L)=\varnothing$. By the spectral theory, there exists a family of projections $\left\{E_{n}=\left(\cdot, f^{(n)}\right) f^{(n)}: n \geq 0\right\}$ such that $P_{t}=\sum_{n \geq 0} \mathrm{e}^{-\lambda_{n} t} E_{n}$. Then,

$$
\begin{aligned}
\left(P_{t} f, g\right) & =\int P_{t} f(x) g(x) \pi(\mathrm{d} x) \\
& =\int \sum_{n \geq 0} \mathrm{e}^{-\lambda_{n} t}\left(f, f^{(n)}\right) f^{(n)}(x) g(x) \pi(\mathrm{d} x) \\
& =\iint \sum_{n \geq 0} \mathrm{e}^{-\lambda_{n} t} f(y) f^{(n)}(y) f^{(n)}(x) g(x) \pi(\mathrm{d} x) \pi(\mathrm{d} y) \\
& =\iint f(y) g(x) p(t, x, y) \pi(\mathrm{d} x) \pi(\mathrm{d} y) .
\end{aligned}
$$

Thus, $p(t, x, y)=\sum_{n \geq 0} \mathrm{e}^{-\lambda_{n} t} f^{(n)}(y) f^{(n)}(x)$, a.e. $\pi \otimes \pi$ and $f^{(0)}=1$, which further yields

$$
\begin{aligned}
d(y, y) & =\int_{0}^{\infty}\left[\sum_{n \geq 0} \mathrm{e}^{-\lambda_{n} t}\left(f^{(n)}(y)\right)^{2}-1\right] \mathrm{d} t \\
& =\int_{0}^{\infty} \sum_{n \geq 1} \mathrm{e}^{-\lambda_{n} t}\left(f^{(n)}(y)\right)^{2} \mathrm{~d} t \\
& =\sum_{n \geq 1} \frac{1}{\lambda_{n}}\left(f^{(n)}(y)\right)^{2} .
\end{aligned}
$$

Combining this with Lemma 2.1, we obtain

$$
T=\int d(y, y) \pi(\mathrm{d} y)=\int \sum_{n \geq 1} \frac{1}{\lambda_{n}}\left(f^{(n)}(y)\right)^{2} \pi(\mathrm{d} y)=\sum_{n \geq 1} \frac{1}{\lambda_{n}} .
$$

\subsection{Strong ergodicity}

It is well known that $T$ can be associated with the strong ergodicity for Markov processes (cf. [1, Chapter 4], [13]). In this subsection we investigate the relationship between $T$ and the rate of the strong ergodicity. Recall that a Markov process $X_{t}$ is said to be strongly ergodic if there is an $\varepsilon>0$ such that

$$
\sup _{x \in \mathbb{R}_{+}}\|P(t, x, \cdot)-\pi\|_{\mathrm{var}}=O\left(\mathrm{e}^{-\varepsilon t}\right) \quad \text { as } t \rightarrow \infty .
$$

Define the (exponential) convergence rate by

$$
\begin{aligned}
\alpha:=\sup \{\varepsilon>0: \text { there exists a constant } C>0, \\
\text { such that } \left.\sup _{x \in \mathbb{R}_{+}}\|P(t, x, \cdot)-\pi\|_{\text {var }} \leq C \mathrm{e}^{-\varepsilon t}, t>0\right\} .
\end{aligned}
$$

It is easy to see that

$$
\alpha=-\sup _{t>0} \frac{1}{t} \sup _{\|f\|_{\infty} \leq 1} \log \left\|P_{t} f-\pi(f)\right\|_{\infty},
$$

where the right-hand side term is well defined by a similar argument as in [9, Section 2]. So, when $\alpha>0$, it is equivalent to the fact that the spectral radius of $P_{t}-\pi$ in $L^{\infty}(\pi)$ is less than 1 
for any $t>0$ (see [20] for the corresponding results for Markov chains). Moreover, the rate $\alpha$ is quite different from $\lambda_{1}$, the spectral gap of $L$ in $L^{2}(\pi)$, which is related to the exponentially ergodic rate in the $L^{2}$-sense. In the following conclusion we present the estimates of $\alpha$ in terms of the eigenvalues in $L^{2}(\pi)$.

Theorem 2.1. Assume that $\infty$ is an entrance boundary. Then we have

$$
\lambda_{1}^{-1} \leq \alpha^{-1} \leq\left[\sum_{n \geq 1}\left(1+f^{(n)^{2}}(0)\right) \lambda_{n}^{-1}\right] \wedge\left[64 \sum_{n \geq 1} \lambda_{n}^{-1}\right] .
$$

Remark 2.1. (a) Mao [13, Proposition 3.2] gave a sufficient condition for the first inequality becoming equality.

(b) The second inequality indicates that $T<\infty$ admits the strong ergodicity.

Proof of Theorem 2.1. The first inequality in (2.4) comes from [13, Proposition 3.2]. For the second inequality, we want to prove the following inequality first:

$$
\alpha^{-1} \leq 64 T
$$

The main idea is essentially due to [1, Lemma 15, Chapter 4] for Markov chains. The key proof is sketched in Appendix A as a completion.

On the other hand, by [15, Theorem 3], we know that $\alpha \geq \delta^{-1}$, where

$$
\delta:=\int_{0}^{\infty} \mathrm{e}^{-c(y)} \mathrm{d} y \int_{y}^{\infty} a(z)^{-1} \mathrm{e}^{c(z)} \mathrm{d} z .
$$

Recall that $S=\int \mu([x, \infty)) \mathrm{d} s(x)$ defined in (1.2). It is then easy to see that $\delta=S$ and

$$
\alpha^{-1} \leq S
$$

We now turn to prove that

$$
S=T+d(0,0)
$$

Recall that $T=(1 / Z) \int \mu((0, t]) \mu([t, \infty)) \mathrm{d} s(t)$. Comparing this to the expressions for $T$ and $S$, we have the following identity:

$$
T=S-\frac{1}{Z} \int_{0}^{\infty} \mu((x, \infty))^{2} \mathrm{~d} s(x) .
$$

In addition, as $\mathbb{E}_{x} H_{0}=\int_{0}^{x} \mu((t, \infty)) \mathrm{d} s(t)$, and by Lemma 2.1, we obtain

$$
\frac{1}{Z} \int_{0}^{\infty} \mu((x, \infty))^{2} \mathrm{~d} s(x)=\int_{0}^{\infty} \mathbb{E}_{x} H_{0} \pi(\mathrm{d} x)=\int_{0}^{\infty}(p(t, 0,0)-1) \mathrm{d} t=d(0,0),
$$

which implies (2.7). Therefore, by (2.5), (2.6), and (2.7), we arrive at

$$
\alpha^{-1} \leq(64 T) \wedge(T+d(0,0)) .
$$

Thus, it suffices to find the spectral expressions for $T$ and $d(0,0)$. Since

$$
d(0,0)=\int_{0}^{\infty}\left(\sum_{n \geq 0} \mathrm{e}^{-\lambda_{n} t}\left(f^{(n)}(0)\right)^{2}-1\right) \mathrm{d} t=\sum_{n \geq 1}\left(f^{(n)}(0)\right)^{2} \frac{1}{\lambda_{n}},
$$

we obtain the second inequality by substituting expressions (2.3) and (2.9) into (2.8). 
Note that in [1], $\alpha^{-1}$ has upper bound 66T. Properly choosing the parameters in our proof leads to the better estimate (see Appendix A for details).

For the ergodic process, i.e. $\mu((0, \infty))<\infty$ and $s(\infty)=\infty$, the following conclusion illustrates the relationship among $T$, eigenvalues of $-L$, and the strong ergodicity.

Theorem 2.2. For the one-dimensional ergodic diffusion process $\left(X_{t}\right)$ generated by $L$, the following statements are equivalent:

(1) the process is strongly ergodic;

(2) $S:=\int \mu([x, \infty)) \mathrm{d} s(x)<\infty$;

(3) $T<\infty$;

(4) $\sigma_{\mathrm{ess}}(L)=\varnothing$ and $\sum_{n \geq 1} \lambda_{n}^{-1}<\infty$.

Proof. We first remark that the equivalence between (1) and (2) is due to Mao [12]. As shown in the proof of Lemma 2.1, (2) is equivalent to (3). So, we need to show that (3) is equivalent to (4). If $T<\infty$ then by the fact that (2) is equivalent to (3), $\infty$ is the entrance boundary. Thus, by Theorem 1.1, (3) implies (4) directly. Conversely, it is easy to see that (4) will imply (3) from the proof of Theorem 1.1.

As an application, we study some distances which are used to estimate the convergence rate of $P_{t}$ towards equilibrium. Due to Theorem 2.2, and with a similar procedure as in [15, Theorem 4.1], we obtain the following corollary.

Corollary 2.1. For the ergodic diffusion process $\left(X_{t}\right)$ generated by $L$, define

$$
d_{p, q}\left(P_{t}, \pi\right)=\left\{\int \pi(\mathrm{d} x)\left(\int \pi(\mathrm{d} y)|p(t, x, y)-1|^{p}\right)^{q / p}\right\}^{1 / q}, \quad p, q \in[1, \infty] .
$$

If $\int_{0}^{\infty} d_{2,2}\left(P_{t}, \pi\right)^{2} \mathrm{~d} t<\infty$ then $\lim _{t \rightarrow \infty} d_{1, \infty}\left(P_{t}, \pi\right)=0$.

Proof. By making full use of the expression $d_{2,2}\left(P_{t}, \pi\right)$, we obtain

$$
\begin{aligned}
d_{2,2}\left(P_{t}, \pi\right)^{2} & =\int_{0}^{\infty} \pi(\mathrm{d} x) \int_{0}^{\infty}(p(t, x, y)-1)^{2} \pi(\mathrm{d} y) \\
& =\int_{0}^{\infty} \pi(\mathrm{d} x)\left(\int_{0}^{\infty} p^{2}(t, x, y) \pi(\mathrm{d} y)-1\right) \\
& =\int_{0}^{\infty} p^{2}(t, x, y) \pi(\mathrm{d} y) \pi(\mathrm{d} x)-1 \\
& =\int_{0}^{\infty}(p(2 t, x, x)-1) \pi(\mathrm{d} x) .
\end{aligned}
$$

It follows that $\int_{0}^{\infty} d_{2,2}\left(P_{t}, \pi\right)^{2} \mathrm{~d} t=T / 2<\infty$ by Lemma 2.1. On the other hand, we have

$$
d_{1, \infty}\left(P_{t}, \pi\right)=\sup _{x} \int_{0}^{\infty}|p(t, x, y)-1| \pi(\mathrm{d} y)=\sup _{x}\|P(t, x, \cdot)-\pi(\cdot)\|_{\mathrm{var}} .
$$

Since $T<\infty$, the process is strongly ergodic. Thus, $\lim _{t \rightarrow \infty} d_{1, \infty}\left(P_{t}, \pi\right)=0$. 


\section{Exit boundary}

In the section we study the eigentime identity for the minimal diffusion process with $\infty$ as the exit boundary.

\subsection{Proof of Theorem 1.2}

Recall that $\left(X_{t}\right)$ is the minimal diffusion process generated by $L$. Define

$$
p(t, x, y)=\frac{P^{\min }(t, x, \mathrm{~d} y)}{\mu(\mathrm{d} y)},
$$

where $P^{\min }(t, x, A)=P_{x}\left(X_{t} \in A ; t<\zeta\right)$. Define the Green function $G=(g(x, y) ; x, y \in$ $\mathbb{R}_{+}$) by

$$
g(x, y):=\int_{0}^{\infty} p(t, x, y) \mathrm{d} t \text { for all } x, y \in \mathbb{R}_{+} .
$$

Denote the trace of $G$ by $\operatorname{tr}(G):=\int g(x, x) \mu(\mathrm{d} x)$. It is well known that if a process is transient, i.e. $\mu((0, \infty))=\infty$ and $s(\infty)=\int_{0}^{\infty} \mathrm{e}^{-c(u)} \mathrm{d} u<\infty$, then $g(x, y)<\infty$ for all $x, y \in \mathbb{R}_{+}$.

Lemma 3.1. For the minimal diffusion process $\left(X_{t}\right)_{t \geq 0}$ with $\infty$ the exit boundary, $\operatorname{tr}(G)=$ $\mathbb{E}_{0}(\zeta)=R<\infty$

Before moving on, it is necessary to recall some useful notation concerning hitting times. It is well known that the following equation (cf. [17]) is the Laplace transform of the hitting time $H_{y}:$ for all $x, y \in \mathbb{R}_{+}$and $\lambda \geq 0$,

$$
\mathbb{E}_{x}\left(\exp \left(-\lambda H_{y}\right)\right)= \begin{cases}\frac{\Phi_{\lambda,-}(x)}{\Phi_{\lambda,-}(y)} & \text { if } x<y \\ \frac{\Phi_{\lambda,+}(x)}{\Phi_{\lambda,+}(y)} & \text { if } x>y\end{cases}
$$

for functions $\Phi_{\lambda, \pm}$ (with $\Phi_{\lambda,-}$ increasing and $\Phi_{\lambda,+}$ decreasing) determined uniquely up to constant factors as a pair of increasing and decreasing nonnegative solutions $\Phi$ of the differential equation

$$
L \Phi=\lambda \Phi,
$$

subject to appropriate boundary conditions $\left(\Phi^{\prime}(0)=0\right.$ for $\Phi_{\lambda,-} ; \Phi(\infty)=0$ for $\left.\Phi_{\lambda,+}\right)$; see [7, pp. 128-130]. This equation leads us to prove Lemma 3.1.

Proof of Lemma 3.1. Since the process is transient, then $g(x, y)=\int_{0}^{\infty} p(t, x, y) \mathrm{d} t<\infty$. The corresponding general Green function (see, e.g. [7], [18]) is

$$
G_{\lambda}(x, y):=\int_{0}^{\infty} \mathrm{e}^{-\lambda t} p(t, x, y) \mathrm{d} t=\omega_{\lambda}^{-1} \Phi_{\lambda,-}(x \wedge y) \Phi_{\lambda,+}(x \vee y),
$$

where the Wronskian $\omega_{\lambda}:=\left(\Phi_{\lambda,-}^{\prime}(y) \Phi_{\lambda,+}(y)-\Phi_{\lambda,-}(y) \Phi_{\lambda,+}^{\prime}(y)\right) / s^{\prime}(y)$ depends only on $\lambda$ but not on $y$. It is easy to see that

$$
\frac{G_{\lambda}(x, y)}{G_{\lambda}(y, y)}=\frac{\Phi_{\lambda,-}(x \wedge y) \Phi_{\lambda,+}(x \vee y)}{\Phi_{\lambda,+}(y) \Phi_{\lambda,+}(y)}= \begin{cases}\frac{\Phi_{\lambda,+}(x)}{\Phi_{\lambda,+}(y)}, & x>y, \\ \frac{\Phi_{\lambda,-}(x)}{\Phi_{\lambda,-}(y)}, & x<y .\end{cases}
$$


Recall that $H_{y}:=\inf \left\{t: X_{t}=y, t<\zeta\right\}$. It is known from [17] that

$$
\mathbb{E}_{x}\left[\exp \left(-\lambda H_{y}\right)\right]= \begin{cases}\frac{\Phi_{\lambda,+}(x)}{\Phi_{\lambda,+}(y)}, & x>y, \\ \frac{\Phi_{\lambda,-}(x)}{\Phi_{\lambda,-}(y)}, & x<y,\end{cases}
$$

which together with (3.1) implies that

$$
\frac{G_{\lambda}(x, y)}{G_{\lambda}(y, y)}=\mathbb{E}_{x}\left[\exp \left(-\lambda H_{y}\right)\right] .
$$

By letting $\lambda \rightarrow 0$, we obtain $\lim _{\lambda \rightarrow 0} \mathbb{E}_{x}\left(\exp \left(-\lambda H_{y}\right)\right)=\mathbb{P}_{x}\left(H_{y}<\infty\right)$. Thus, $g(x, y)=$ $g(y, y) \mathbb{P}_{x}\left(H_{y}<\infty\right)$, where $\mathbb{P}_{x}$ stands for the probability taken for the underlying process starting from $x$. Since $\mathbb{P}_{0}\left(H_{y}<\infty\right)=1$, we further obtain

$$
\int g(0, y) \mu(\mathrm{d} y)=\int g(y, y) \mu(\mathrm{d} y):=\operatorname{tr}(G) .
$$

On the other hand,

$$
\int_{0}^{\infty} p(t, 0, y) \mu(\mathrm{d} y)=\int_{0}^{\infty} P(t, 0, \mathrm{~d} y ; t<\zeta)=\mathbb{P}_{0}(\zeta>t) .
$$

Then, we obtain

$$
\int g(0, y) \mu(\mathrm{d} y)=\iint_{0}^{\infty} p(t, 0, y) \mathrm{d} t \mu(\mathrm{d} y)=\int_{0}^{+\infty} \mathbb{P}_{0}(\zeta>t) \mathrm{d} t=\mathbb{E}_{0} \zeta .
$$

Therefore, $\mathbb{E}_{0} \zeta=\operatorname{tr}(G)=R<\infty$.

Proof of Theorem 1.2. The essential idea is similar to the case with $\infty$ as the entrance boundary, and we just sketch the proof. First, by a similar discussion as in Lemma 2.2, we know that if $\operatorname{tr}(G)<\infty$ then $P_{t}$ is a Hilbert-Schmidt operator for all $t>0$. Thus, $\sigma_{\text {ess }}(L)=\varnothing$. Then, due to the spectral expression of $p(t, x, y), \operatorname{tr}(G)$ can be represented as $\sum_{n=1}^{\infty} \lambda_{n}^{-1}$, which leads to the finial conclusion by using Lemma 3.1 .

\subsection{Uniform decay}

Similar to the entrance case, the eigentime identity for $\infty$ as the exit boundary can be related to the uniform decay (see, e.g. [16]), which is the case where

$$
\sup _{x \in \mathbb{R}_{+}}\|P(t, x, \cdot)\|_{\mathrm{var}}=O\left(\mathrm{e}^{-\varepsilon t}\right), \quad t \rightarrow \infty .
$$

Define (exponential) uniform decay rate by

$$
\begin{aligned}
& \beta:=\sup \{\varepsilon>0: \text { there exists a constant } c>0, \\
& \text { such that } \left.\sup _{x \in \mathbb{R}^{+}}\|P(t, x, \cdot)\|_{\text {var }} \leq c \mathrm{e}^{-\varepsilon t} \text { for all } t>0\right\} .
\end{aligned}
$$

In the following theorem we present the estimate of $\beta$ by using the spectrum in $L^{2}(\mu)$. 
Theorem 3.1. For the one-dimensional minimal diffusion process $\left(X_{t}\right)$ with the exit boundary $\infty$, we have

$$
\lambda_{1}^{-1} \leq \beta^{-1} \leq \sum_{n \geq 1} \lambda_{n}^{-1} .
$$

Proof. The proof is similar to that of [14, Theorem 1.8] for Markov chains, and we omit it here.

We end this section by collecting a series of explicit equations for transient processes, which are equivalent to the rule of the uniform decay.

Theorem 3.2. For the one-dimensional minimal diffusion process $\left(X_{t}\right)$ generated by $L$, the following statements are equivalent:

(1) the process is uniformly decaying;

(2) $R:=\int \mu([0, x)) \mathrm{d} s(x)<\infty$;

(3) $\operatorname{tr}(G)<\infty$;

(4) $\mathbb{E}_{0} \zeta<\infty$;

(5) $\sigma_{\mathrm{ess}}(L)=\varnothing$ and $\sum_{n \geq 1} \lambda_{n}^{-1}<\infty$.

\section{Appendix A.}

Proof of ' $\alpha^{-1} \leq 64 \sum_{n \geq 1} \lambda_{n}^{-1}$ ' in Theorem 2.1. Recall some basic notation to measure the deviation from stationarity at time $t$ first. Let

$$
d(t):=\sup _{x}\left\|\mathbb{P}_{x}\left(X_{t} \in \cdot\right)-\pi(\cdot)\right\|_{\mathrm{var}} .
$$

Note that

$$
\left\|\mathbb{P}_{x}\left(X_{t} \in \cdot\right)-\pi(\cdot)\right\|=2 \sup _{A \in \mathcal{B}}\left(\mathbb{P}_{x}\left(X_{t} \in A\right)-\pi(A)\right),
$$

where $\mathcal{B}$ are the Borel- $\sigma$ fields. Then, it is easy to see that

$$
\frac{1}{2} d(s+t) \leq \frac{1}{2} d(s) \times \frac{1}{2} d(t)
$$

We also define some useful parameters,

$$
\begin{aligned}
& \tau_{1}:=\inf \left\{t: \frac{1}{2} d(t) \leq \mathrm{e}^{-1}\right\}, \\
& \tau_{2}:=\sup _{x, y} \int_{0}^{\infty}\left|\mathbb{E}_{x} H_{z}-\mathbb{E}_{y} H_{z}\right| \pi(\mathrm{d} z), \\
& \tau_{3}:=\sup _{x \in \mathbb{R}_{+}, A \in \mathcal{B}} \pi(A) \mathbb{E}_{x} H_{A} .
\end{aligned}
$$

The first parameter is to formalize the idea of 'time to approach stationarity from the worst starting place'. The second parameter measures the variability of mean hitting times as the starting place varies. The final parameter is regarded as the ratio of $\mathbb{E}_{x} H_{A}$, the diffusion process mean hitting time on $A$, to $1 / \pi(A)$. By [1, Lemma 14, Chapter 4], we have $\tau_{3} \leq \tau_{2} \leq 2 T$. If $\tau_{1}<\infty$ then from submultiplicativity it follows that

$$
\frac{1}{2} d(t) \leq \exp \left(1-\frac{t}{\tau_{1}}\right), \quad t \geq 0,
$$


and, hence, $\alpha^{-1} \leq \tau_{1}$. Now, we turn to prove that $\tau_{1} \leq 64 T$. For small $\delta>0$, which will be specified later, let

$$
A:=\left\{x: \mathbb{E}_{\pi} H_{x} \leq \frac{T}{\delta}\right\}
$$

According to the Markov inequality and the definition of $T$, we infer that

$$
\pi\left(A^{c}\right)=\pi\left\{x: \mathbb{E}_{\pi} H_{x}>\frac{T}{\delta}\right\} \leq \frac{\int \mathbb{E}_{\pi} H_{x} \pi(\mathrm{d} x)}{T / \delta}=\frac{T}{T / \delta}=\delta .
$$

For any $x \in \mathbb{R}_{+}$, by the monotonicity of $p(t, x, x)$ about $t$ and $p(t, x, x) \geq 1$, which is explained in the proof of Lemma 2.2, we obtain

$$
\mathbb{E}_{\pi} H_{x}=\int_{0}^{\infty}(p(s, x, x)-1) \mathrm{d} s \geq t(p(t, x, x)-1) \quad \text { for all } t \in \mathbb{R}_{+} .
$$

Thus, for any $y \in A$, we have

$$
p(t, y, y)-1 \leq \frac{\mathbb{E}_{\pi} H_{y}}{t} \leq \frac{T}{\delta t}
$$

which together with the inequality

$$
|p(t+s, x, y)-1| \leq \sqrt{(p(2 t, x, x)-1)(p(2 s, y, y)-1)}
$$

implies that

$$
p(t, x, y) \geq 1-\frac{T}{\delta t}, \quad x, y \in A .
$$

Let $x$ be arbitrary and $z \in A$. For all $0 \leq s \leq u$,

$$
\frac{\mathbb{P}_{x}\left(X_{u+t} \in \mathrm{d} z \mid H_{A}=s\right)}{\pi(\mathrm{d} z)} \geq \inf _{y \in A} \frac{\mathbb{P}_{y}\left(X_{u+t-s} \in \mathrm{d} z\right)}{\pi(\mathrm{d} z)}=\inf _{y \in A} p(u+t-s, y, z) .
$$

Thus,

$$
p(u+t, x, z) \geq\left(1-\frac{T}{\delta t}\right)^{+} \mathbb{P}_{x}\left(H_{A} \leq u\right) .
$$

By using the Markov inequality and the definition of $\tau_{3}$, we conclude that

$$
\mathbb{P}_{x}\left(H_{A}>u\right) \leq \frac{\mathbb{E}_{x} H_{A}}{u} \leq \frac{\tau_{3}}{u \pi(A)} .
$$

Since $\tau_{3} \leq \tau_{2} \leq 2 T$, and by inequalities (A.1) and (A.2) we obtain

$$
p(u+t, x, z) \geq\left(1-\frac{T}{\delta t}\right)^{+}\left(1-\frac{2 T}{u \pi(A)}\right)^{+}=: \eta<1 .
$$

Since $x$ is arbitrary, we have

$$
\begin{aligned}
\frac{1}{2} d(u+t) & =\frac{1}{2} \sup _{x} \int|p(u+t, x, z)-1| \pi(\mathrm{d} z) \\
& =\sup _{x}\left(1-\int 1 \wedge p(u+t, x, z) \pi(\mathrm{d} z)\right)
\end{aligned}
$$




$$
\begin{aligned}
& \leq \sup _{x}\left(1-\int_{A} 1 \wedge p(u+t, x, z) \pi(\mathrm{d} z)\right) \\
& \leq 1-\eta \pi(A) \\
& \leq 1-\left(1-\frac{T}{\delta t}\right)^{+}\left(\pi(A)-\frac{2 T}{u}\right)^{+} \\
& \leq 1-\left(1-\frac{T}{\delta t}\right)^{+}\left(1-\delta-\frac{2 T}{u}\right)^{+} .
\end{aligned}
$$

Taking $t=46 T, u=18 T$, and $\delta=\sqrt{92} / 69$, we obtain the bound $4 \sqrt{23} / 69+37 / 414 \leq \mathrm{e}^{-1}$, i.e. $\tau_{1} \leq 64 T$. Therefore, we have $\alpha^{-1} \leq 64 T$. Note that Aldous and Fill [1] achieved the result $\alpha^{-1} \leq 66 T$ by putting $t=49 T, u=17 T$, and $\delta=1 / 7$. A slight modification in our proof makes a better estimation.

\section{Acknowledgements}

The authors would like to thank the anonymous reviewers for their valuable comments and suggestions. Research supported in part by the start-up research fund supplied by Zhejiang University of Technology (grant number 109007329), 985 Project, 973 Project (grant number 2011CB808000), NSFC (National Natural Science Foundation of China)(grant number 11131003), SRFDP (Specialized Research Fund for the Doctoral Program of Higher Education) (grant number 20100003110005) and the Fundamental Research Funds for the Central Universities.

\section{References}

[1] Aldous, D. J. AND Fill, J. A. (2002). Reversible Markov chains and random walks on graphs. Unpublished manuscript. Available at http://www.stat.berkeley.edu/ aldous/RWG/book.html.

[2] Borodin, A. N. And Salminen, P. (2002). Handbook of Brownian Motion-Facts and Formulae, 2nd edn. Birkhäuser, Basel.

[3] Cheng, L.-J. and Mao, Y.-H. (2013). Passage time distribution for one-dimensional diffusion processes. Preprint.

[4] Douglas, R. G. (1998). Banach Algebra Techniques in Operator Theory (Graduate Texts Math. 179). Springer, New York.

[5] Feller, W. (1952). The parabolic differential equations and the associated semi-groups of transformations. Ann. Math. 55, 468-519.

[6] Feller, W. (1954). Diffusion processes in one dimension. Trans. Amer. Math. Soc. 77, 1-31.

[7] Itô, K. And Mckean, H. P. Jr. (1965). Diffusion Processes and Their Sample Paths. Spinger, Berlin.

[8] Karlin, S. and Taylor, H. M. (1981). A Second Course in Stochastic Processes. Academic Press, New York.

[9] Liggett, T. M. (1989). Exponential $L_{2}$ convergence of attractive reversible nearest particle systems. Ann. Prob. 17, 403-432.

[10] Mandl, P. (1968). Analytical Treatmeat of One-Dimensional Markov Processes. Springer, Berlin.

[11] Mao, Y.-H. (2002). Deviation kernels for one-dimensional diffusion processes. Preprint. Available at http://math.bnu.edu.cn/probab/Workshop2002/Preprints/MaoYH.pdf.

[12] MaO, Y.-H. (2002). Strong ergodicity for Markov processes by coupling methods. J. Appl. Prob. 39, $839-852$.

[13] Mao, Y.-H. (2004). The eigentime identity for continuous-time ergodic Markov chains. J. Appl. Prob. 41, 1071-1080.

[14] Maо, Y. H. (2006). Eigentime identity for trainsient Markov chains. J. Math. Anal. Appl. 315, $415-424$.

[15] Mao, Y. H. (2006). Some new results on strong ergodicity. Front. Math. China 1, 105-109.

[16] Mao, Y.-H. and OuYang, S.-X. (2006). Strong ergodicity and uniform decay for Markov processes. Math. Appl. 19, 580-586. 
[17] Pitman, J. And Yor, M. (2003). Hitting, occupation and inverse local times of one-dimensional diffusions: martingale and excursion approaches. Bernoulli 9, 1-24.

[18] Reed, M. And Simon, B. (1972). Methods of Modern Mathematical Physics. I. Functional Analysis. Academic Press, New York.

[19] Reed, M. And Simon, B. (1975). Methods of Modern Mathematical Physics. II. Fourier Analysis, SelfAdjointness. Academic Press, New York.

[20] Wu, L. (2004). Essential spectral radius for Markov semigroups. I. Discrete time case. Prob. Theory Relat. Fields 128, 255-321. 